\title{
Confronting Stellar Evolution Models for Active and Inactive Solar-Type Stars: The Triple System V1061 Cygni
}

\author{
Guillermo Torres ${ }^{1}$, Claud H. Sandberg Lacy ${ }^{2}$, Laurence A. \\ Marschall $^{3}$, Holly A. Sheets ${ }^{4}$, and Jeff A. Mader ${ }^{5}$ \\ ${ }^{1}$ Harvard-Smithsonian Center for Astrophysics, 60 Garden St., Cambridge, MA 02138 \\ e-mail: gtorres@cfa.harvard.edu \\ ${ }^{2}$ Dept. of Physics, University of Arkansas, Fayetteville, AR 72701 \\ ${ }^{3}$ Dept. of Physics, Gettysburg College, 300 North Washington Street, Gettysburg, PA 17325 \\ ${ }^{4}$ Dept. of Physics \& Astronomy, Dartmouth College, Hanover, NH 03755 \\ ${ }^{5}$ W.M. Keck Observatory, Kamuela, HI 96743
}

\begin{abstract}
.
We present spectroscopic and photometric observations of the chromospherically active (X-ray strong) eclipsing binary V1061 Cyg $(P=2.35$ days $)$ showing that it is in reality a hierarchical triple system. We combine these observations with Hipparcos intermediate data (abscissa residuals) to derive the outer orbit with a period of $15.8 \mathrm{yr}$. We determine accurate values for the masses, radii, and effective temperatures of the eclipsing binary components, as well as for the mass and temperature of the third star. For the primary we obtain $M=1.282 \pm 0.015 \mathrm{M}_{\odot}$, $R=1.615 \pm 0.017 \mathrm{R}_{\odot}, T_{\text {eff }}=6180 \pm 100 \mathrm{~K}$, for the secondary $M=0.9315 \pm 0.0068 \mathrm{M}_{\odot}$, $R=0.974 \pm 0.020 \mathrm{R}_{\odot}, T_{\text {eff }}=5300 \pm 150 \mathrm{~K}$, and for the tertiary $M=0.925 \pm 0.036 \mathrm{M}_{\odot}$ and $T_{\text {eff }}=5670 \pm 100 \mathrm{~K}$. Current stellar evolution models agree well with the properties of the primary star, but show a large discrepancy in the radius of the secondary in the sense that the observed value is about $10 \%$ larger than predicted (a $5 \sigma$ effect). We also find the secondary temperature to be $\sim 200 \mathrm{~K}$ cooler than indicated by the models. These discrepancies are quite remarkable considering that the secondary is only $7 \%$ less massive than the Sun, which is the calibration point of all stellar models. Similar differences with theory have been seen before for lower mass stars. We identify chromospheric activity as the likely cause of the effect. Inactive stars agree very well with the models, while active ones such as the secondary of V1061 Cyg appear systematically too large and too cool. Both of these differences are understood in terms of the effects of magnetic fields commonly associated with chromospheric activity.
\end{abstract}

Keywords. binaries: eclipsing, binaries: spectroscopic, stars: individual (V1061 Cyg), techniques: radial velocities

\section{Introduction}

V1061 Cygni is a rather neglected eclipsing binary with a period of 2.35 days. It was originally thought to be an example of the rare class of "Cool Algols" (Popper 1996), a group of semi-detached systems of which less than a dozen members are known. We present here new photometric and spectroscopic observations that not only show the system to be well detached, but also reveal it to be a hierarchical triple with all three stars visible in the spectra. Furthermore, the properties of the three components turn out to be very useful for testing models of stellar evolution. In recent years compelling evidence has been presented of discrepancies in the predictions of theory for stars under a solar mass. In particular, models for such objects have been found to underestimate their radii by at least $10 \%$ (a deviation far greater than the typical uncertainties), and to overestimate 
their effective temperatures by some $200 \mathrm{~K}$. The reasons for these discrepancies have never been clear, but chromospheric activity has been mentioned as a possibility, among several others, given that low-mass stars often show obvious signs of activity including spots, Ca II H and K emission, X-ray emission, etc.

In this work we combine our radial-velocity measurements for the three stars with astrometric measurements from the Hipparcos mission and with accurate measurements of the times of eclipse. This has allowed us to determine the elements of the inner and outer orbits. The velocities along with the light curves then yield the physical properties of the stars to high accuracy. A comparison with current stellar evolution models and with the properties of other similar stars has provided important insight into the problem mentioned above.

\section{Observations}

Spectroscopic observations were obtained at the Harvard-Smithsonian Center for Astrophysics (CfA) over a period of more than 17 years, and are shown in the next section. Radial velocities for the three components of the system were derived using an extension of the two-dimensional cross-correlation algorithm TODCOR to 3 dimensions (Zucker et al. 1995). Light curves were obtained at the University of Arkansas (URSA telescope, $V$ band) and at Gettysburg College $(B V R I)$. They are displayed in Figure 1, along with our best fits using the Nelson-Davis-Etzel model (EBOP code) as described by Etzel (1981) and Popper \& Etzel (1981).
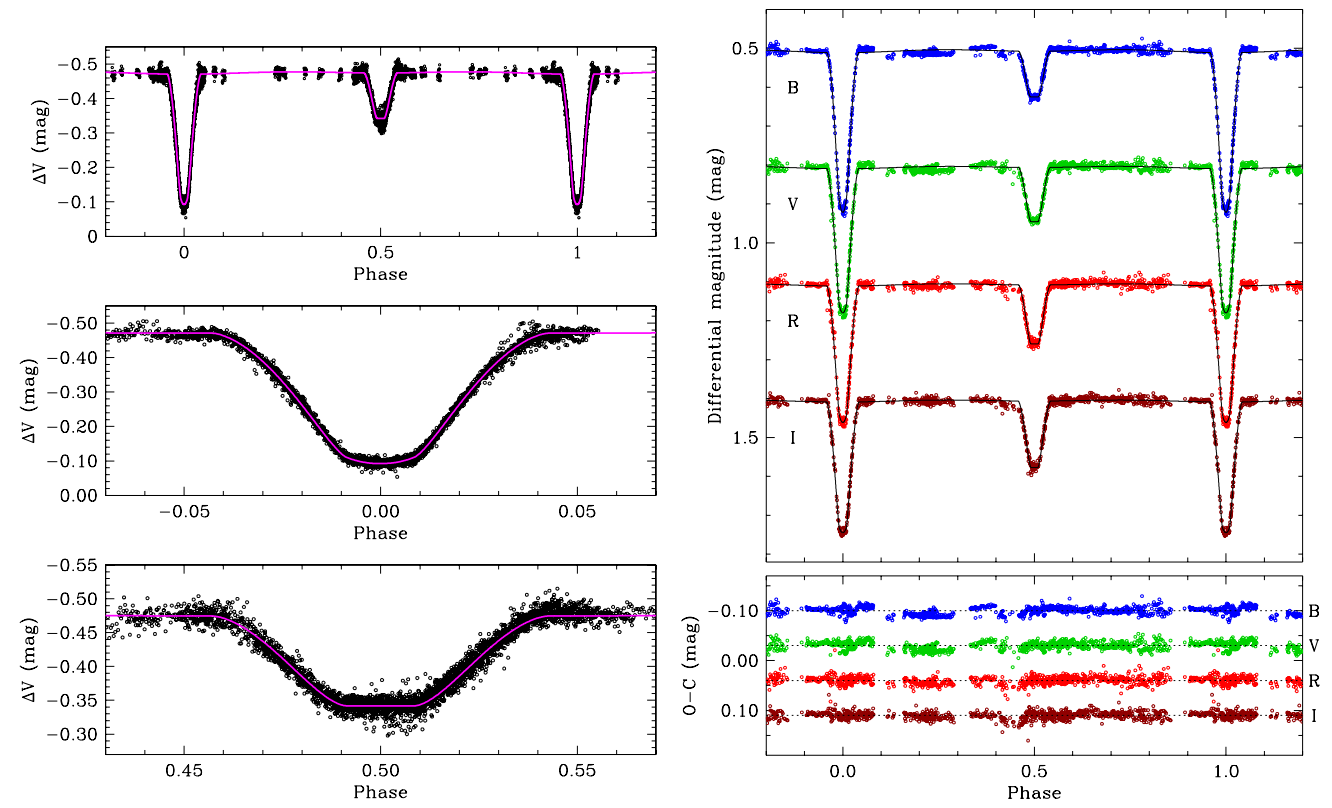

Figure 1. Light curves from the URSA telescope ( $V$ band, left) and Gettysburg College ( $B V R I)$. Distortions possibly due to spots are visible in the residuals of the latter (bottom panel), near phase 0.45 .

V1061 Cyg was classified by Hipparcos as a "Variability-Induced Mover", indicating a perturbation in the astrometric motion of the center of light due to the presence of the third star. We incorporate the intermediate astrometric data (abscissa residuals) into our 
triple orbital solution, along with our radial velocities for the three stars and some 70 years' worth of times of eclipse from this work and from the literature.

\section{Orbital solutions}

We solved simultaneously for the elements of the inner and outer orbits, as well as the third-body effect on the times of eclipse. Figure 2 is a representation of the spectroscopic orbit of the inner eclipsing pair, after subtracting the motion of its center-of-mass in the outer orbit. This orbit is circular and has a period of $2.34665473 \pm 0.00000035$ days.

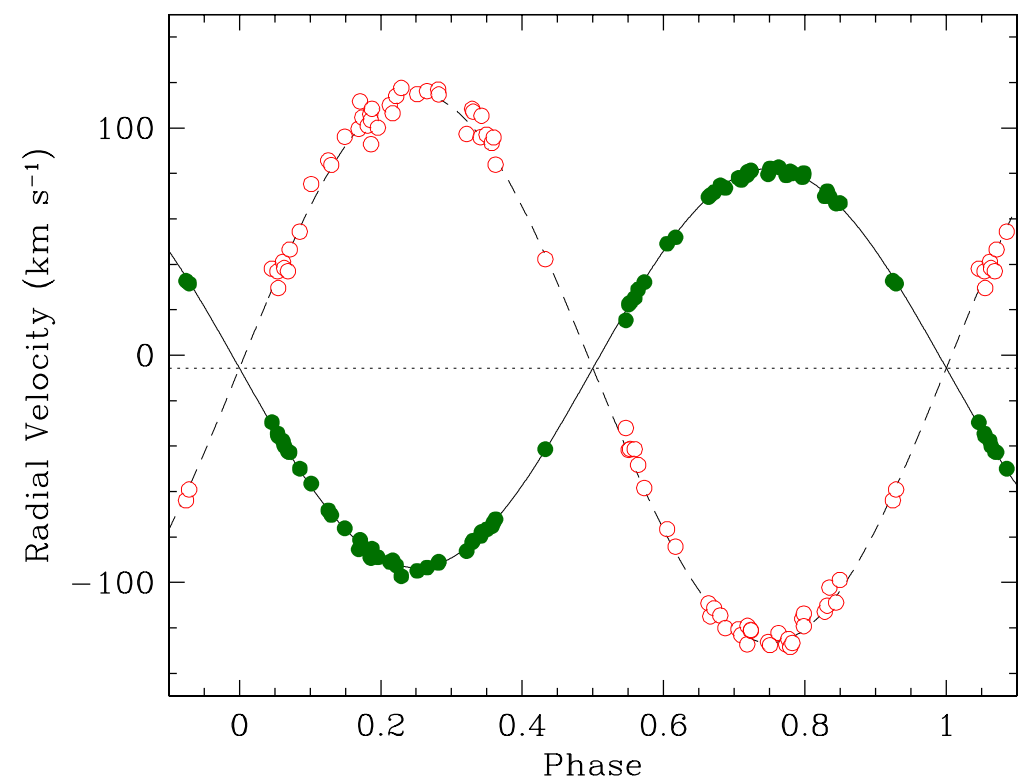

Figure 2. Velocity measurements for the inner pair along with our computed orbit. The dotted line represents the center-of-mass velocity of the triple. Phase 0.0 corresponds to the time of primary eclipse.

In Figure 3, top panel, we show the radial-velocity curve from our solution for the outer orbit, with a period of $15.84 \pm 0.21$ years and an eccentricity $e=0.469 \pm 0.032$. The velocities for the primary and secondary (stars Aa and Ab) have been corrected for the motion in the inner orbit, and are averaged together for this figure (blue crosses). The bottom panel displays the $O-C$ residuals from the most recent times of eclipse, which show clearly the modulation due to the distant tertiary (star B) and provide the strongest constraint on the outer period. The location of the Hipparcos observations in time is shown by the green crosses.

Figure 4 is a representation on the plane of the sky of the orbit of the center of light as observed by Hipparcos (eclipsing binary + third star) around the center of mass of the triple system. The semimajor axis of this orbit is $9.55 \pm 0.54$ mas. The one-dimensional Hipparcos observations are shown schematically. Because these measurements are onedimensional in nature, the exact location of each observation on the plane of the sky cannot be shown graphically. The filled circles represent the predicted location on the computed orbit. The dotted lines connecting to each filled circle indicate the scanning direction of the Hipparcos satellite for each measurement, and show which side of the orbit the residual is on. The short line segments at the end of and perpendicular to the dotted lines indicate the direction along which the actual observation lies, although the 


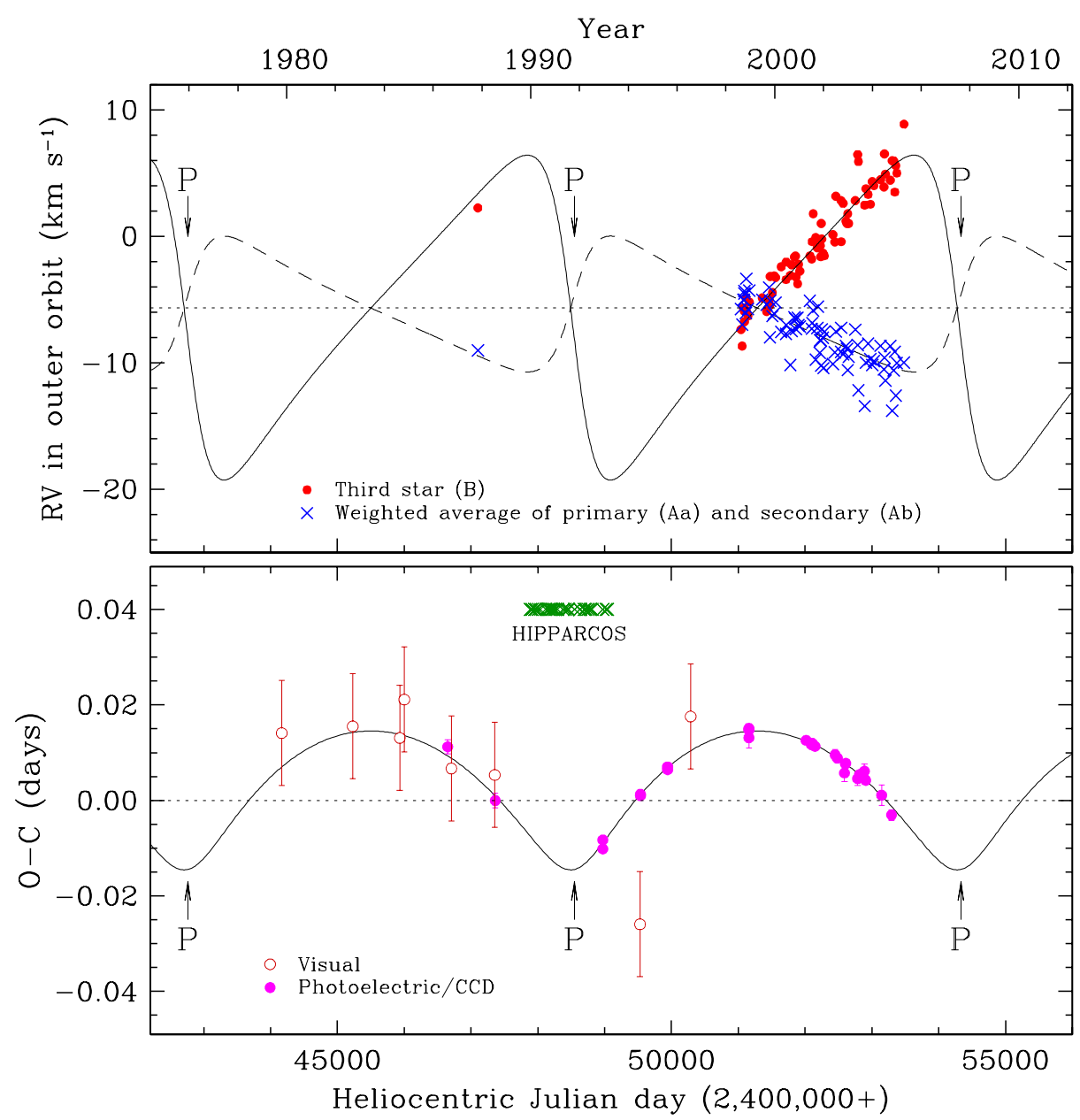

Figure 3. Radial velocities for V1061 Cyg in the outer orbit, and $O-C$ eclipse timing residuals as a function of time. The curves represent our best-fit model that uses velocities simultaneously with the eclipse timings and Hipparcos observations. Top: Velocities for the third star (filled circles) and the center-of-mass of the inner binary (crosses). The latter is computed at each date from the weighted average of the two stars, after removing the motion in the inner orbit. The center-of-mass velocity is indicated with the dotted line. Bottom: $O-C$ diagram of the more recent eclipse timings based on a linear ephemeris. Open circles are for visual measurements, and filled circles for photoelectric/CCD measurements. The dates of the Hipparcos observations are indicated, and happen to cover periastron passage (arrows).

precise location is undetermined. Occasionally more than one measurement was taken along the same scanning direction, in which case two or more short line segments appear on the same dotted lines.

\section{Comparison with models}

Table 1 gives accurate values for the physical properties of the primary and secondary of V1061 Cyg derived from our analysis of the spectroscopic and photometric observations. The masses and radii are good to better than $2 \%$. The comparison with current stellar evolution models by Yi et al. (2001) shown in Figure 5 indicates good agreement for the primary star for an age of $3.4 \mathrm{Gyr}$ and solar composition, but the secondary radius is $10 \%$ 


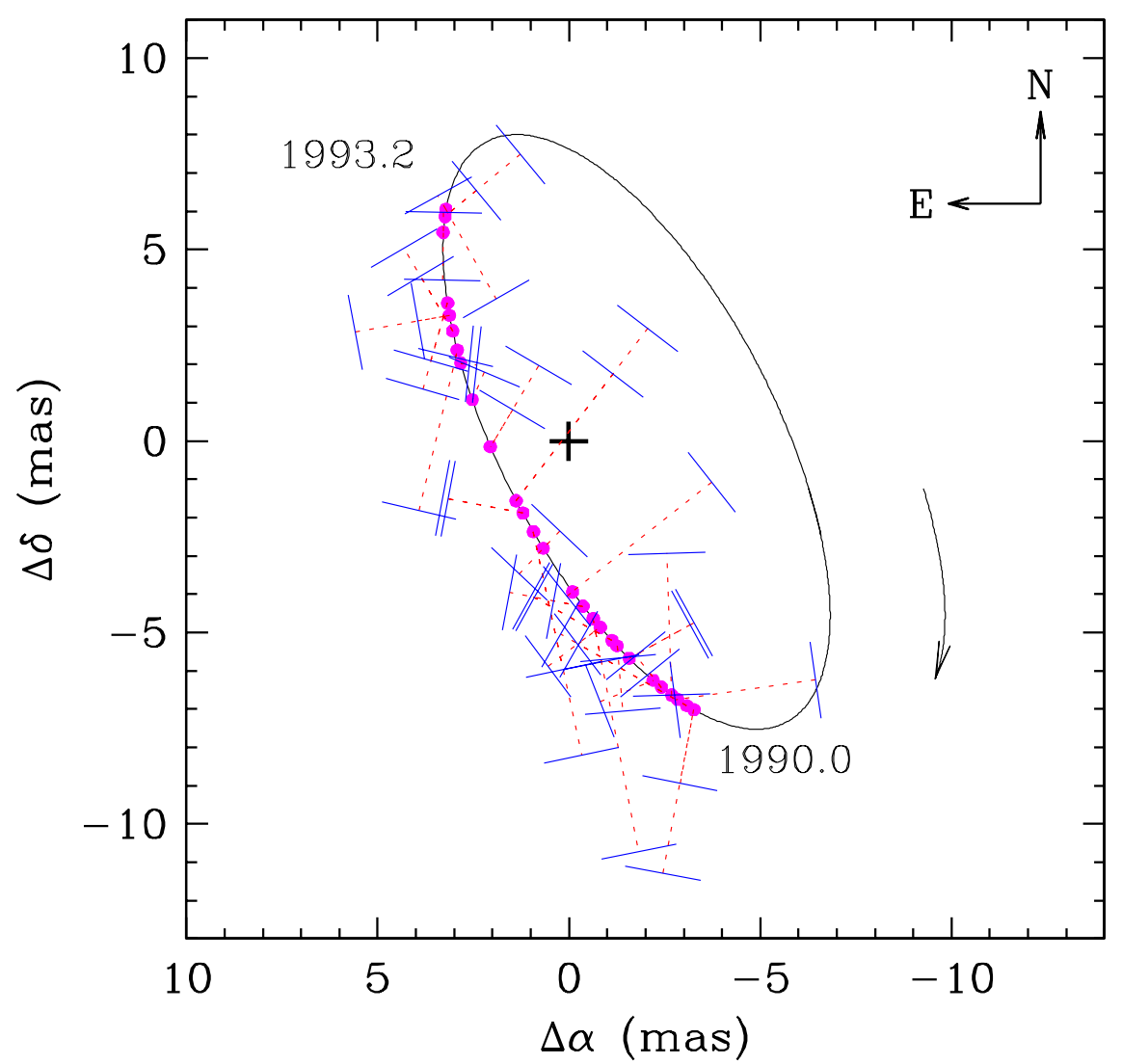

Figure 4. Path of the center of light of V1061 Cyg on the plane of the sky, along with the Hipparcos observations (abscissa residuals), after subtracting the parallactic and proper-motion contributions. The plus sign represents the center of mass of the triple, and the direction of motion in the orbit (retrograde) is indicated by the arrow. See text for an explanation of the graphical representation of these measurements.

Table 1. Physical parameters for V1061 Cyg.

\begin{tabular}{lccc}
\hline \multicolumn{1}{c}{ Parameter } & Primary & Secondary & Tertiary \\
\hline Mass $\left(\mathrm{M}_{\odot}\right)$ & $1.283 \pm 0.016$ & $0.9322 \pm 0.0071$ & $0.926 \pm 0.037$ \\
Radius $\left(\mathrm{R}_{\odot}\right)$ & $1.604 \pm 0.016$ & $0.964 \pm 0.014$ & $\ldots$ \\
$\log g$ & $4.136 \pm 0.010$ & $4.439 \pm 0.013$ & $\ldots$ \\
Temperature $(\mathrm{K})$ & $6180 \pm 100$ & $5300 \pm 150$ & $5670 \pm 150$ \\
$\log L / L_{\odot}$ & $0.527 \pm 0.029$ & $-0.181 \pm 0.051$ & $\ldots$ \\
$M_{V}(\mathrm{mag})$ & $3.459 \pm 0.073$ & $5.503 \pm 0.082$ & $5.30 \pm 0.19$ \\
$v \sin i\left(\mathrm{~km} \mathrm{~s}^{-1}\right)$ & $36 \pm 2$ & $20 \pm 3$ & $2 \pm 3$ \\
$v_{\text {sync } \sin i\left(\mathrm{~km} \mathrm{~s}^{-1}\right)}$ & $34.6 \pm 0.4$ & $20.8 \pm 0.3$ & $\ldots$ \\
Distance $(\mathrm{pc})$ & $165.6 \pm 5.5$ & $165.6 \pm 5.5$ & $165.6 \pm 5.5$ \\
$m-M(\mathrm{mag})$ & $6.095 \pm 0.072$ & $6.095 \pm 0.072$ & $6.095 \pm 0.072$ \\
\hline
\end{tabular}

larger than predicted (a $5 \sigma$ discrepancy). Experiments show that no adjustment in the age or metallicity is able to remove the disagreement. Since the secondary brightness is well matched by the models, it follows that the predicted effective temperature must also be off ( $\sim 200 \mathrm{~K}$ too high). These disagreements are quite remarkable in that V1061 Cyg B is only $7 \%$ less massive than the Sun, which is the calibration point of all stellar models. 

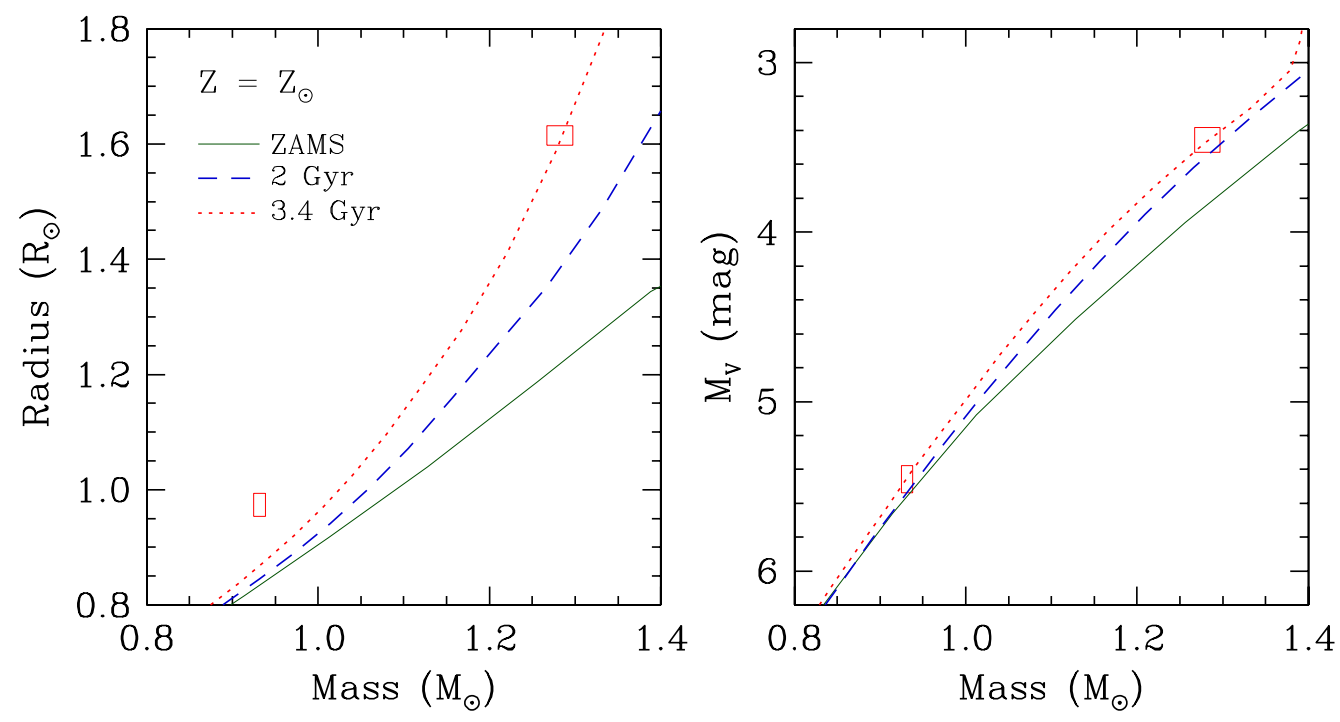

Figure 5. Comparison between the observed properties (error boxes) of the primary and secondary of V1061 Cyg (mass, radius, absolute visual magnitude) and model isochrones by Yi et al. (2001) and Demarque et al. (2004) for solar metallicity and three different ages, as labeled.

\section{Understanding the discrepancies}

Three other eclipsing binaries with one component being in the same mass range as V1061 Cyg B (within 3\%) have accurately determined parameters from the literature: RW Lac, FL Lyr, and HS Aur (Lacy et al. 2005, Popper 1986, Andersen 1991). As shown below, the secondary in the FL Lyr system exhibits the same radius discrepancy as V1061 Cyg B, while both stars in the other two systems are well fit by the models, within the errors (see Figure 6). Why?

As it turns out, both V1061 Cyg and FL Lyr show clear signs of activity: their orbital periods are short $\left(\sim 2\right.$ days), and the stars are rapidly rotating $\left(v \sin i>20 \mathrm{~km} \mathrm{~s}^{-1}\right)$ due to tidal forces that keep them synchronized with the orbital motion; they show intrinsic variations in their light curves (spottedness); they are also strong X-ray sources (as detected by ROSAT), with $\log L_{\mathrm{X}}>30 \mathrm{erg} \mathrm{s}^{-1}$. On the other hand RW Lac and HS Aur, which are well fit by the models, have long orbital periods ( $\sim 10$ days), slow rotations $\left(v \sin i<5 \mathrm{~km} \mathrm{~s}^{-1}\right)$, and display no $\mathrm{X}$ rays. This indicates a connection between the activity and the discrepancies noted above: Active stars appear larger and cooler than normal.

\section{Discussion}

This connection between activity and the radius discrepancies has been suspected before for stars under a solar mass (e.g., Popper 1997, Clausen et al. 1999, Torres \& Ribas 2002, Ribas 2003, López-Morales \& Ribas 2005). In fact, Mullan \& MacDonald (2001) showed a similar effect in a study of active and inactive $\mathrm{M}$ dwarfs, but without precise knowledge of the stellar radii or masses. As far as we can tell this is the first time it has been shown clearly for stars so close to the mass of the Sun with accurately determined masses and radii. Theory provides an explanation for the effect in terms of the magnetic fields commonly associated with chromospheric activity: they tend to inhibit convective heat transport, and consequently the star grows larger in order to radiate away 

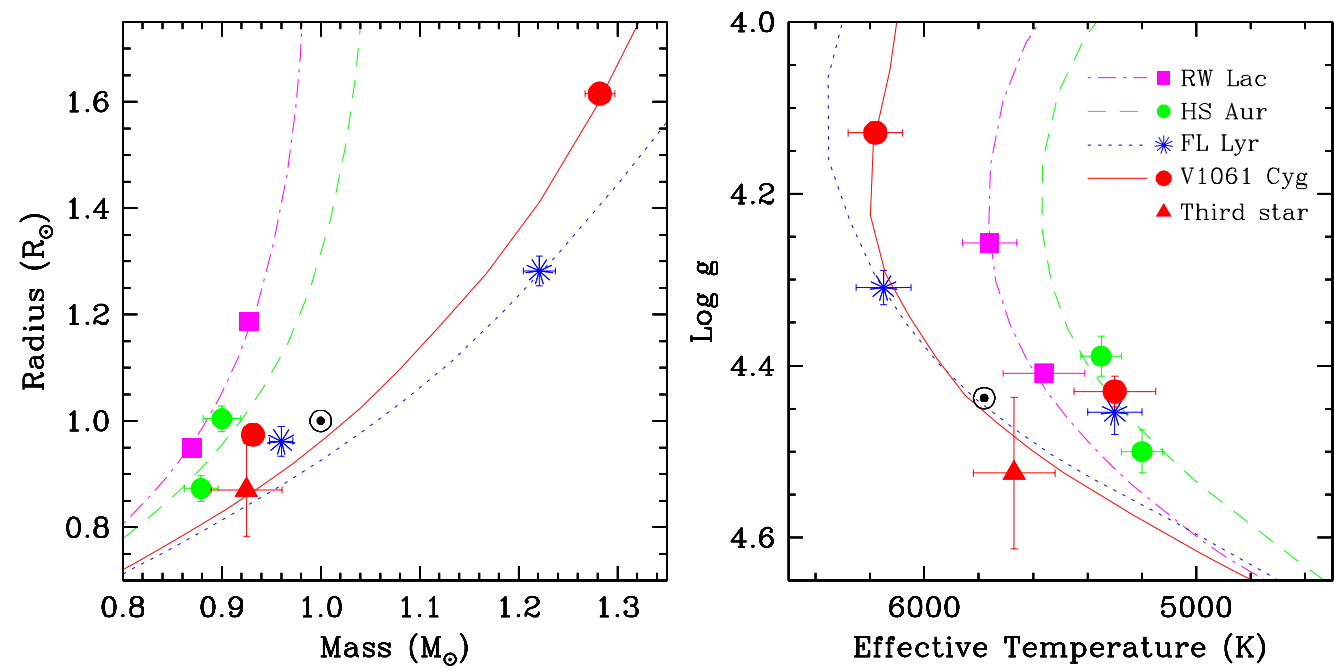

Figure 6. Properties of V1061 Cyg and three other eclipsing binaries having one star closely matching V1061 Cyg Ab in mass, compared against the Yonsei-Yale models by Yi et al. (2001) and Demarque et al. (2004). For each binary system the model isochrone best fitting all observed properties is shown: $Z=0.0118$ and age $=10.7 \mathrm{Gyr}$ for RW Lac, $Z=0.024$ and age $=10.5$ Gyr for HS Aur, $Z=0.026$ and age $=2.4 \mathrm{Gyr}$ for FL Lyr, and $Z=Z_{\odot}$ and age $=3.4$ Gyr for V1061 Cyg. The secondary star in FL Lyr is significantly larger than predicted, as is V1061 Cyg Ab (see text). The Sun $(\odot)$ is also shown for reference, along with the third component of V1061 Cyg (triangle).

the same amount of internal energy. This also explains why stellar evolution models with lower convective efficiency (smaller mixing length parameter) appear to show less of a discrepancy when compared with the observations.

The third star in V1061 Cyg provides an ideal check since it has the same mass as the secondary but is inactive. Although less precise, its radius (inferred from the radiative properties) and effective temperature are consistent with being normal for a star of its mass (see Figure 6), supporting our main conclusion.

For full details of our study we refer the reader to our publication (Torres et al. 2006).

\section{Acknowledgements}

We thank J.M. Kreiner for providing unpublished times of eclipse for the binary, as well as R. Neuhäuser and B. Stelzer for assistance with the X-ray observations. GT acknowledges partial support for this work from NSF grant AST-0406183 and NASA's MASSIF SIM Key Project (BLF57-04). LM and HS were supported by Gettysburg College and the Delaware Space Grant Consortium. Additional thanks go to Peter Mack and Gary Hummer for technical support at the Gettysburg College Observatory, as well as to J. Andersen, P. Berlind, M. Calkins, J. Caruso, D.W. Latham, R.P. Stefanik, and J. Zajac for their efforts at the CfA to obtain the majority of the spectroscopic observations for V1061 Cyg used in this work. 


\section{References}

Andersen, J. 1991, A\&A Rev., 3, 91

Clausen, J.V., Baraffe, I., Claret, A., \& VandenBerg, D.A. 1999a, in Theory and Tests of Convection in Stellar Structure, eds. A. Giménez, E.F. Guinan, \& B. Montesinos, ASP Conf. Ser. 173 (San Francisco: ASP), 265

Demarque, P., Woo, J.-H., Kim, Y.-C., \& Yi, S.K. 2004, ApJS, 155, 667

Etzel, P.B. 1981, in Photometric and Spectroscopic Binary Systems, ed. E.B. Carling \& Z. Kopal (NATO ASI Ser. C., 69) (Dordrecht: Reidel), 111

Lacy, C.H.S., Torres, G., \& Claret, A., \& Vaz, L.P.R. 2005, AJ, 130, 2838

Lejeune, Th., Cuisinier, F., \& Buser, R. 1998, A\&A, 130, 65

López-Morales, M. \& Ribas, I. 2005, ApJ, 631, 1120

Mullan, D.J., \& MacDonald, J. 2001, ApJ, 559, 353

Popper, D.M. 1996, ApJS, 106, 133

Popper, D.M. 1997, AJ, 114, 1195

Popper, D.M., \& Etzel, P.B. 1981, AJ, 86, 102

Popper, D.M., Lacy, C.H., Frueh, M.L., \& Turner, A., E. 1986, AJ, 91, 383

Ribas, I. 2003, $A \& A$ A, 398, 239

Torres, G., Lacy, C.H., Marschall, L.A., Sheets, H.A., \& Mader, J.A. 2006, ApJ, 640, 1018

Torres, G., \& Ribas, I. 2002, ApJ, 567, 1140

Yi, S.K., Demarque, P., Kim, Y.-C., Lee, Y.-W., Ree, C.H., Lejeune, T., \& Barnes, S. 2001, ApJS, 136, 417

Zucker, S., Torres, G., \& Mazeh, T. 1995, ApJ, 452, 863 